\title{
A Novel Method for Determining Microbial Kinetics
}

\author{
Anne M. Talkington, Floyd L. Inman III and Leonard D. Holmes \\ Sartorius Stedim-Biotechnology Laboratory, Biotechnology Research and Training Center, University of North Carolina at \\ Pembroke, Pembroke, North Carolina 28372, USA
}

Received: January 04, 2013 / Accepted: March 21, 2013 / Published: August 30, 2013.

\begin{abstract}
Understanding microbial growth is essential to any research conducted in the fields of microbiology and biotechnology. Current methods of determining growth characteristics of microbes involve subjective graphical interpretations of linearized logarithmic data. Reducing error in logistical data decreases disparity between graphical and analytical predictions of microbial characteristics. In this study, a method has been developed to calculate the kinetics of microbial characteristics utilizing a modified Maclaurin series. Convergence of this series approaches the true kinetic value of microbial characteristics to include specific growth rates. In this research, a modified Maclaurin series is used to evaluate microbial kinetics in comparison to graphical determinations.
\end{abstract}

Key words: Microbial kinetics, Maclaurin series, microbial growth models, series convergence.

\section{Introduction}

Research in microbial biotechnology relies on the study of microbial kinetics [1]. Microbial kinetics can be approximated by different models. These models include the: (1) Monod, (2) Gompertz, (3) Contois, (4) Baranyi-Roberts and (5) logistic equations [2-5]. A common method of determining growth rates is the graphical approach whereby the specific growth rate is calculated from the logarithmic transformation of the raw data [6]. Through this transformation process, the exponential phase of growth is linearized where graphical interpretations are used to approximate points within the exponential growth phase. Once identified, a line of best fit is generated. The slope of this line defines the microbial growth rate for the specified conditions [7].

The Maclaurin series, a specific Taylor series polynomial expansion, is a function that converges to an equivalent function where the center of convergence is zero [8]. Based on its successive higher-order derivatives, this function is defined as the

Corresponding author: Leonard D. Holmes, Ph.D., associate professor, research fields: fermentation technology, applied microbiology and biochemistry. E-mail: len.holmes@uncp.edu. sum of its terms. A notable formulation of the Maclaurin series is the exponential model, which converges for all real values across its domain. Like Malthusian models, the Maclaurin exponential model can apply to microbial growth. The Maclaurin model has been modified with additional terms and is suitable for both logistic and semi-logistic growth. Present applications of the Maclaurin series suffer from truncation error when it is evaluated only to the first two terms. By expanding the series, error is reduced and the precision of the series is increased for evaluating microbial characteristics.

Traditional logistic curves are symmetrical, however, any disparity from symmetry represents the lack of idealness of the culture [8]. With reduced truncation, this series can serve as a method for calculating the rates of symmetrical and nonsymmetrical growth characteristics through its convergent properties. The application of the Maclaurin series as a method to determine growth associated kinetics is significant as it reduces subjectivity and dependence on the graphical method. The introduction of an alternating series may account for culturing parameters (substrate availability, $\mathrm{pH}$, bacterial density, etc.) that may promote or limit 
microbial growth [9]. As the term number approaches infinity, the value for each term will approach zero. Furthermore, as the series converges, successively smaller overestimates and underestimates of the true value are obtained [8]. Therefore, an Alternating series which is shown in Fig. 1 models the culturing environment and generates an extremely close approximation of the true kinetic value.

\section{Methods}

In calculating microbial kinetics, the value of the inflection point is determined to pinpoint the shift from exponential increase to exponential decrease. The inflection point is defined as the maximum value of the signal prior to the beginning of the exponential decrease. It is identified at the maximum rate of increase, where logistic data changes from exponentially increasing to exponentially decreasing. Previously, the calculation of inflection points involved derivative analysis of symmetrical sets [8]; however, the development of a more convenient formula allowed for simpler calculations. The inflection point (a value of $P$ ) was calculated as the lower limit $+1 / 2$ (higher limit - lower limit) as seen in Fig. 2a. For nonsymmetrical data sets, the inflection point was determined by dissecting the initial curve into two symmetrical curves. Therefore, the inflection point becomes evident as it is the intersection of the two curves, as shown in Fig. 2b. All evaluations of the modified series for each data set were performed utilizing Texas Instruments TI-84 and TI-89 graphing calculators.

The modified series exhibits the properties of both alternating and Maclaurin series. It maintains the

$$
\begin{gathered}
\frac{d P}{d t}=\sum_{n=0}^{\infty} \frac{(-1)^{n}\left(n ! 2^{n}\right) r P^{n+1}}{M^{n}(n+1) !} \\
\frac{d P}{d t}=r P-\frac{2 r P^{2}}{M(2 !)}+\frac{8 r P^{3}}{M^{2}(3 !)}-\frac{48 r P^{4}}{M^{3}(4 !)}+\frac{384 r P^{5}}{M^{4}(5 !)}-\frac{3840 r P^{6}}{M^{5}(6 !)}+\cdots+\frac{(-1)^{n}\left(n ! 2^{n}\right) r P^{n+1}}{M^{n}(n+1) !}
\end{gathered}
$$

Fig. 1 Modified alternating Maclaurin series: simplified form (top) $\&$ expanded form (bottom). Where: $n=$ term number, $P$ $=$ measured signal, $M=$ highest signal value, $r=$ rate of increase of signal or growth, and $t=$ time.
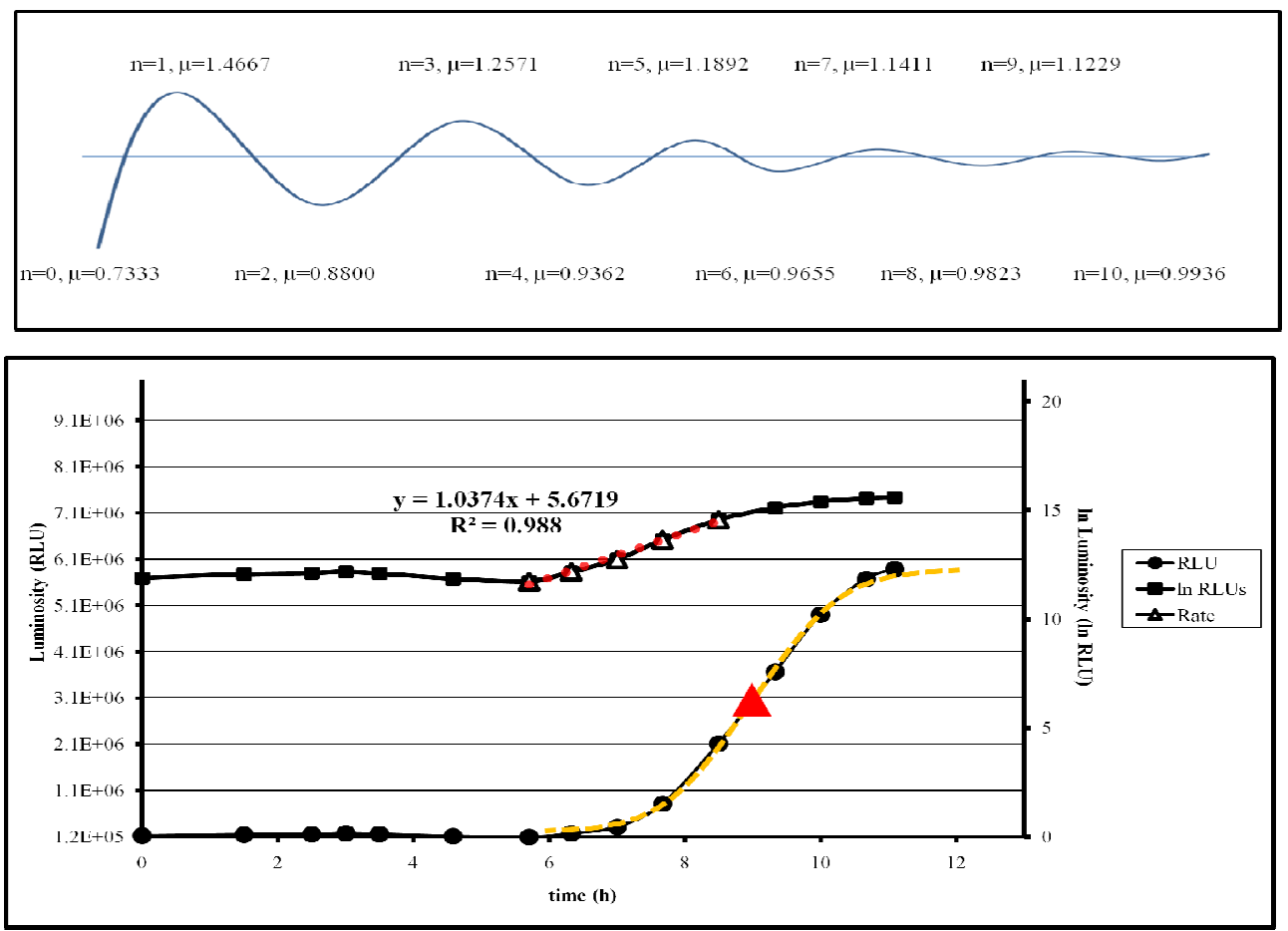

(a) 

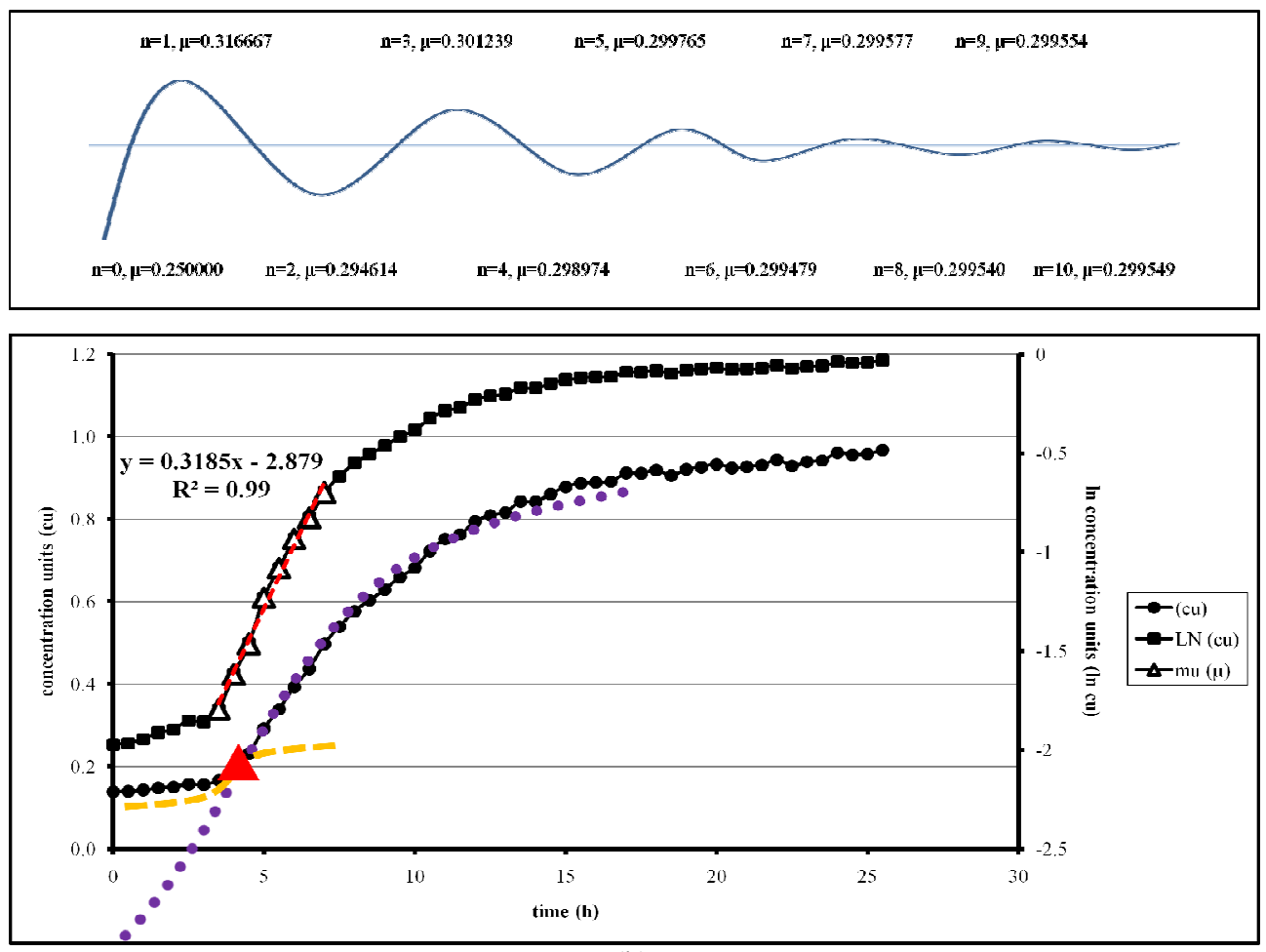

(b)

Fig. 2 Comparison between graphical methods and the modified Maclaurin series for luminosity and concentration units. (a) Symmetrical luminosity data (RLU) as a function of time, the graphical method (above) determines the rate of luminosity to be $1.0 \mathrm{~h}^{-1}$ as the Maclaurin series (below) predicts the rate $(r)$ to be $0.99 \mathrm{~h}^{-1}$; (b) Nonsymmetrical infrared absorbance data (cu) as a function of time, the graphical method (above) determines the microbial growth rate $(\mu)$ to be $0.32 \mathrm{~h}^{-1}$, while the modified Maclaurin (below) estimates $\mu$ to be $0.30 h^{-1}$.

essential exponential component as identified by Malthus et al. [8] and Gause [9]. Furthermore, it is not dependent on derivatives as other traditional Maclaurin series. This series takes the form of a differential equation and the derivative has been resolved to a coefficient based on the term number. The addition of the alternating term (-1n) ensures that each term will oscillate between overestimates and underestimates as the series approaches convergence as supported by Leibniz's Theorem. Its interval of convergence is determined by the Ratio Test [8]. Therefore, this series can serve as a model to solve for microbial kinetics. An evaluation of the series to the first ten terms predictably yields values within $10 \%$ error, whereas other methods of determining kinetics may not show a consistent range of error.

\section{Results and Discussion}

The alternating series is a suitable model for logistic data that incorporates factors of microbial growth. Unstable environmental factors (e.g. pH, temperature) could offset the expected logistic symmetry. When compared, the graphical and Maclaurin approximations should agree within $10 \%$. Any error higher than $10 \%$ may indicate experimental error. Population growth in limited conditions may be logistic [10]. Therefore, a Maclaurin logistic model was implemented to fit calculations of microbial growth. The legitimacy of the modified Maclaurin series is supported by Leibniz's Theorem. The Ratio Test has determined the interval of convergence to be the domain of time $(t)$ including all points from 0 to the inflection point. Application of the series lends itself to a practical, efficient method of modeling microbial characteristics with consistency. Logistic curves are not unit specific; therefore, the same results and principles should apply with any measure of signal (biomass, turbidity, etc.). The oscillating 
properties of the alternating series suggest the potential for a sinusoidal function worthy of further exploration. Perhaps this function can serve as other methods for predicting error, determining convergence, or complementing the Maclaurin series. The establishment of this approach has the potential to facilitate the analysis of microbial characteristics. The authors are presently collecting additional microbial data and creating a software program that can be used to evaluate microbial kinetics.

\section{Conclusion}

The potential for adopting the modified Maclaurin series as a standard laboratory technique for data analysis is promising. Its dependence on physical parameters, represented in part by the error measurement, rather than on the researcher's interpretation of the linearized exponential phase, allows for consistent and comparable results between studies. This research takes into account the expected limited growth pattern. In either a continuous fed-batch culture, or a severely limited scenario such as a batch culture, the calculation for the point of inflection would need to reflect where the point of inflection would be in such scenarios. The inflection point is the point in which time and the population is no longer replicating exponentially under the initial environmental conditions. This practice would not only represent previous methods, but also allows consistency between researchers. The proposed modified model analyzes microbial growth as a series of interactions between population size and carrying capacity. The physical interpretation of the model thus shows that the increase in population size decreases the time needed to achieve carrying capacity. Through this model, it is assumed that deteriorating conditions drive the carrying capacity of the culture. Future research involves solving the differential solution for the model that represents the total convergence of the series without truncation restrictions. The solution may be achieved if an infinite number of terms can be used to account for all physical parameters (substrate, $\mathrm{pH}$, etc.). Research continues to examine the properties of the series as well as the properties that result from truncating the model after a determined number of terms. Understanding the Maclaurin series model allows for both a rigorous interpretation and efficient calculations of microbial growth.

\section{Acknowledgements}

The authors thank the University of North Carolina at Pembroke's (UNCP) Office of the Provost and Academic Affairs; UNCP Department of Chemistry and Physics; North Carolina Biotechnology Center and Robeson Farm Bureau for partial financial support.

\section{References}

[1] T.D. Brock, Microbial growth rates in nature, Bacteriological Reviews 35 (1) (1971) 39-58.

[2] J. Monod, The growth of bacterial cultures, Annual Reviews of Microbiology 3 (1949) 371-394.

[3] M.H. Zwietering, I. Jongenburger, F.M. Rombouts, K.V. Riet, Modeling of the bacterial growth curve, Applied and Environmental Microbiology 56 (6) (1990) 1875-1881.

[4] D.E. Contois, Kinetics of bacterial growth: Relationship between population density and specific growth rate of continuous cultures, Journal of General Microbiology 21 (1959) 40-50.

[5] K. Grijspeerdt, P. Vanrolleghem, Estimating the parameters of the Baranyi model for bacterial growth, Food Microbiology 16 (1999) 593-605.

[6] N.S. Panikov, Microbial Growth Kinetics, Chapman \& Hall, London, 1995.

[7] S. Perni, P.W. Andrew, G. Shama, Estimating the maximum specific growth rate from microbial growth curves: Definition is everything, Food Microbiology 22 (2005) 491-495.

[8] F.D. Demana, R.L. Finney, D. Kennedy, B.K. Waits, Calculus: Graphical, Numerical, Algebraic, Prentice Hall, Upper Saddle River, NJ, 2003.

[9] G.F. Gause, Struggle for Existence, Williams and Wilkins, Baltimore, MD, 1934.

[10] G.F. Gause, Experimental studies on the struggle for existence. I. Mixed populations of two species of yeast, Journal of Experimental Biology 9 (4) (1932) 389-402. 